\title{
Relação entre chuva de sementes e estrutura florestal em remanescentes de Floresta Atlântica no Sul do Brasil
}

\author{
Elivane Salete Capellesso', Kellin Luana Scrovonski², Elisabete Maria Zanin ${ }^{3}$ \\ \& Tanise Luisa Sausen ${ }^{4}$
}

\begin{abstract}
' Universidade Federal do Paraná, Laboratório de Ecologia Vegetal, Departamento de Ciências Biológicas Av. Cel. Francisco H. dos Santos, 100, Curitiba, CEP: 81530-000. elivanesc@gmail.com

2 Universidade Regional Integrada do Alto Uruguai e das Missões, Laboratório de Ecologia e Sistemática Vegetal. Departamento de Ciências Biológicas. Av. Sete de Setembro, 1621, CEP 99709-510, Erechim, RS. kellinscrovonski@hotmail.com ${ }_{3}^{3}$ Universidade Regional Integrada do Alto Uruguai e das Missões, Laboratório de Geoprocessamento e Planejamento Ambiental, Departamento de Ciências Biológicas. Av. Sete de Setembro, 1621, CEP 99709-510, Erechim, RS. emz@uri.com.br

${ }_{4}^{4}$ Universidade Regional Integrada do Alto Uruguai e das Missões, Laboratório de Ecologia e Sistemática Vegetal, Departamento de Ciências Biológicas. Av. Sete de Setembro, 1621, CEP 99709-510, Erechim, RS. tasausen@uricer.edu.br
\end{abstract}

Recebido em 18.1.2016

Aceito em 30.VII.2018

DOI 10.21826/2446-8231201873209

RESUMO - A relação entre a chuva de sementes e a estrutura do estrato arbóreo adulto e regenerante foi avaliada em dois pequenos fragmentos de Mata Atlântica no Sul do Brasil. Foram realizadas coletas mensais da chuva de sementes e a caracterização fitossociológica do estrato arbóreo adulto e regenerante. $\mathrm{O}$ fragmento de floresta nativa 1 (FN1) apresentou relação positiva entre o estrato arbóreo adulto, regenerante e a chuva de sementes. No fragmento de floresta nativa 2 (FN2), o estrato arbóreo adulto não apresentou relação com a chuva de sementes. Adicionalmente, no FN1 observou-se maior riqueza e diversidade na chuva de sementes, porém com menor abundância de indivíduos. As áreas não apresentaram diferença entre a riqueza e abundância de sementes e também não foram observadas diferenças entre as estratégias de dispersão. Os resultados indicam que a estrutura dos estratos e a composição da chuva de sementes são semelhantes em pequenos fragmentos florestais.

Palavras-chave: diversidade, estratégias de dispersão, riqueza de espécies

ABSTRACT - Relationship between seed rain and forest structure in Atlantic Forest remnants in southern Brazil. The relationship between seed rain and adult and regenerating tree structure was evaluated in two small fragments of the Atlantic rainforest in southern Brazil. Monthly seed rain collection and phytosociological characterization of the adult and regenerating tree stratum were performed. Native forest fragment 1 (FN1) showed a positive relationship between adult and regenerating stratum and seed rain. In the native forest fragment 2 (FN2), the adult stratum was not related with seed rain. Additionally, FN1 had higher seed rain richness and diversity, but lower abundance of individuals. The areas did not have a difference between richness and abundance, and also no differences were found between the strategies of dispersion. The results indicate that adult and regenerating tree stratum and seed rain composition are similar in small forest fragments.

Keywords: diversity, dispersal strategies, species richness

\section{INTRODUÇÃO}

A Mata Atlântica é considerada um dos hotspots de diversidade mundial (Myers et al. 2000), restando, atualmente, cerca de $16 \%$ da área original, distribuída em pequenos fragmentos florestais (SOS Mata Atlântica, 2015). O processo de fragmentação influencia a composição da comunidade vegetal (Laurance et al. 2006) e diminui a sua diversidade (Fahrig 2003), com efeitos sobre a disponibilidade de frutos e sementes (Terborgh 1986). A chuva de sementes se refere à quantidade de sementes que chegam ao solo ao longo de um período de tempo (Hardesty \& Parker 2003). O estabelecimento de espécies arbóreas após os eventos de dispersão das sementes é crucial para os processos de sucessão e regeneração da vegetação (Clark et al. 1998, Vieira \& Scariot 2006).
O estabelecimento de plântulas durante o processo de regeneração é influenciado pela fenologia e pela presença e abundância de dispersores (Martínez-Garza et al. 2011), estando diretamente relacionado com a produção e densidade da chuva de sementes e a dinâmica do banco de sementes no solo (Simpson 1989). O conhecimento sobre a composição da chuva de sementes é fundamental para recomendações de planos de recuperação de áreas degradadas (Pietro-Souza et al. 2014), além de planos de restauração ecológica.

O objetivo deste estudo foi avaliar a relação entre o estrato arbóreo e regenerante e a chuva de sementes em fragmentos com tamanho pequeno e baixa conectividade pertencentes ao Bioma Mata Atlântica no Sul do Brasil. A questão a ser investigada neste estudo é se nestes fragmentos florestais, a regeneração florestal por meio da chuva de 
sementes será responsável pela formação de uma comunidade semelhante ao observado no estrato arbóreo adulto.

\section{MATERIAL E MÉTODOS}

\section{Área de estudo}

Foram selecionados dois fragmentos de floresta nativa com a presença de riachos de terceira ordem, localizados no sul do Brasil. Um dos fragmentos florestais localizase no município de Barão de Cotegipe (FN 1), entre as coordenadas $27^{\circ} 28^{\prime} 39^{\prime \prime} \mathrm{S} 52^{\circ} 31^{\prime} 45^{\prime \prime} \mathrm{O}$ e $27^{\circ} 39^{\prime} 40^{\prime \prime} \mathrm{S}$ $52^{\circ} 20^{\prime} 24^{\prime \prime} \mathrm{O}$, e no município de Gaurama (FN 2), entre as coordenadas $27^{\circ} 30^{\prime} 21^{\prime \prime} \mathrm{S} 52^{\circ} 11^{\prime} 10^{\prime \prime} \mathrm{O}$ e $27^{\circ} 40^{\prime} 43^{\prime \prime} \mathrm{S}$ $52^{\circ} 02^{\prime} 15^{\prime \prime} \mathrm{O}$. Durante o período experimental observou-se uma média de precipitação anual de $175 \mathrm{~mm}$ e temperaturas médias mensais oscilando entre 13 e $22^{\circ} \mathrm{C}$.

Os fragmentos florestais correspondem a vegetação característica da Floresta Atlântica com Araucária (Oliveira-Filho et al. 2013), apresentando área média de $49 \mathrm{ha}$. A floresta nativa encontra-se bastante fragmentada e degradada, com os maiores índices de vegetação em locais com declividade acentuada do terreno, a pouca profundidade do solo, com a presença de afloramentos rochosos impossibilitando práticas agrícolas em grande escala (Decian et al. 2009).

\section{Coleta e análise de dados}

Para a realização da coleta de dados foram demarcadas 10 unidades amostrais de $10 \times 20 \mathrm{~m}$ em ambos os fragmentos florestais, sendo cinco unidades em cada lado do riacho, totalizando uma área amostral de $200 \mathrm{~m}^{2}$. Para caracterização estrutural do estrato arbóreo adulto, todos os indivíduos com perímetro altura do peito (PAP) a partir de $15 \mathrm{~cm}$ foram amostrados e a altura foi mensurada com medidor laser (DLE 50, Bosch Professional). Para o levantamento do estrato arbóreo regenerante, foi mensurado o diâmetro altura do solo (DAS) de todos os indivíduos a partir de $0,30 \mathrm{~cm}$ de altura até $15 \mathrm{~cm}$ de PAP, conforme Capellesso et al. (2016). O material coletado foi identificado de acordo com literatura específica (Sobral et al. 2013).

Para a amostragem da chuva de sementes foram instalados 10 coletores de madeira de $1 \mathrm{~m}^{2} \mathrm{em}$ cada fragmento florestal, sendo cinco em cada margem do riacho, distantes 10 metros cada um. A coleta das sementes foi realizada mensalmente entre os meses de janeiro a dezembro de 2013. O material foi triado e identificado ao nível de espécie. Para cada fragmento florestal, as espécies amostradas, foram classificadas de acordo com a estratégia de dispersão proposta por Van der Pijl (1982), sendo classificadas como zoocórica, anemocórica e autocórica. A partir da chuva de sementes foi avaliada a densidade de sementes por $\mathrm{m}^{2}\left(1 \mathrm{~m}^{2}\right)$.

A composição florística dos fragmentos florestais foi determinada pelo número de indivíduos, de espécies e famílias e pelo Índice de Diversidade de Shannon. Para avaliar a relação entre o estrato arbóreo adulto e o regenerante em cada área de estudo foi realizado um teste de Mantel.

Para o número de sementes e a riqueza de espécies foi realizado um teste $t$ entre as áreas. Para verificar as diferenças entre as estratégias de dispersão e as áreas de estudo foi realizado uma Análise de Variância One-way (ANOVA). Foi realizada uma análise comparando entre si, a densidade de sementes e a riqueza de espécies, por meio de um teste de qui-quadrado $\left(x^{2}\right)$ para uma amostra independente para cada área e estrato arbóreo.

A relação entre a chuva de sementes com o estrato arbóreo adulto e regenerante de cada fragmento florestal foi avaliada por meio de um teste de Mantel. Todas as análises foram realizadas no ambiente estatístico " $R$ " (Team 2012).

\section{RESULTADOS}

$\mathrm{O}$ fragmento de FN1 apresenta maior número de indivíduos, riqueza de espécies e famílias em ambos os estratos arbóreo adulto e regenerante em relação ao fragmento de Floresta nativa 2 (Tab. 1). FN1 apresenta relação positiva entre o estrato arbóreo adulto e regenerante $(\mathrm{r}=0.501 ; \mathrm{p}=0.002)$, mas este padrão não foi observado para FN2 ( $\mathrm{r}=0.044 ; \mathrm{p}=0.438$ ). A diversidade e equabilidade foram maiores no FN1, indicando que as espécies foram igualmente abundantes (Tab. 1).

As espécies presentes na chuva de sementes do FN2 foram semelhantes às amostradas no FN1 (Tab. 2). Porém, o FN1 apresentou maior riqueza de espécies e diversidade, apesar de uma menor abundância e densidade de sementes (Tab. 3). Não houve diferença quanto ao número de sementes $(\mathrm{t}=1,42 ; \mathrm{df}=21,53 ; \mathrm{p}=0,16)$ e riqueza de espécies $(\mathrm{t}=-0,08 ; \mathrm{df}=21,39 ; \mathrm{p}=0,93)$ entre os fragmentos florestais na chuva de sementes (Fig. 1).

A densidade de sementes não diferiu entre os fragmentos e as estratégias de dispersão $(\mathrm{F}=0,132 ; \mathrm{d}=1$; $\mathrm{p}=0,877$ ) (Tab. 3). A chuva de sementes foi composta, principalmente, por espécies zoocóricas, sendo 17 espécies em Floresta nativa 1 e 14 espécies no Floresta nativa 2. As estratégias de dispersão levando-se em consideração a proporção de indivíduos e espécies apresentaram Diferença em cada fragmento florestal $(\mathrm{p} \leq 0,0001)$, porém não observou-se diferença entre os fragmentos florestais (Tab. 3).

A estrutura do estrato arbóreo adulto e regenerante da Floresta nativa 1 apresenta relação positiva a chuva de sementes $(r=0,27, p=0,05$ e $r=0,30 ; p=0,03)$. Além disso, observou-se que para a Floresta nativa 2, apenas a estrutura do estrato arbóreo regenerante apresenta relação com a chuva de sementes $(r=0,39 ; p=0,02)$, sendo que este padrão não foi observado para o estrato arbóreo adulto $(\mathrm{r}=0,05 ; \mathrm{p}=0,38)$. 
Tabela 1. Abundância de indivíduos $\left(\mathrm{em} 200 \mathrm{~m}^{2}\right)$, riqueza de espécies, riqueza de famílias, índice de diversidade de Shannon Weaver (nats ind. $\left.{ }^{-1}\right) \mathrm{e}$ Equitabilidade de Pielou a partir da caracterização estrutural do estrato arbóreo adulto e regenerante nos fragmentos de Floresta nativa 1 e Floresta nativa 2.

\begin{tabular}{lcccc}
\hline \multirow{2}{*}{ Variáveis } & & FN 1 & \multicolumn{2}{c}{ FN 2 } \\
\cline { 2 - 5 } & Regeneração & Adulto & Adulto & 223 \\
\hline Abundância de ind. & 3221 & 353 & 53 & 219 \\
Riqueza de espécies & 75 & 53 & 23 & 12 \\
Riqueza de famílias & 29 & 33 & 2.755 \\
Índice de diversidade & 3.352 & 0.828 & 0.691 \\
Índice de Equitabilidade & 0.776 & 0.770 & 2.691 \\
\hline
\end{tabular}

Abundância de indivíduos, riqueza de espécies e de famílias calculadas a partir do número de indivíduos total amostrados na área de estudo.

Tabela 2. Lista de espécies, abundância de sementes amostradas na chuva de sementes e estratégias de dispersão das espécies presentes nos fragmentos de Floresta nativa 1 (FN1) e Floresta nativa 2 (FN2).

\begin{tabular}{|c|c|c|c|}
\hline Família/Espécie & $\begin{array}{c}\text { Estratégia de dispersão da } \\
\text { espécie } \\
\end{array}$ & $\begin{array}{l}\text { Abundância sementes no } \\
\text { FN1 }\end{array}$ & $\begin{array}{c}\text { Abundância de sementes } \\
\text { no FN2 } \\
\end{array}$ \\
\hline $\begin{array}{l}\text { Boraginaceae } \\
\text { Cordia americana (L.) Gottshling \& J.E.Mill. }\end{array}$ & Anemocórica & 103 & 2 \\
\hline $\begin{array}{l}\text { Euphorbiaceae } \\
\text { Sapium glandulosum (L.) Morong } \\
\text { Sebastiania brasiliensisSpreng. } \\
\text { S. commersoniana (Baill.) L.B. Sm. \& Downs }\end{array}$ & $\begin{array}{l}\text { Autocórica } \\
\text { Autocórica } \\
\text { Autocórica }\end{array}$ & $\begin{array}{c}1 \\
14 \\
8\end{array}$ & $\begin{array}{c}- \\
10 \\
-\end{array}$ \\
\hline $\begin{array}{l}\text { Fabaceae } \\
\text { Ateleia glazioviana Baill. } \\
\text { Dalbergia frutescens (Vell.) Britton } \\
\text { Parapiptadenia rigida (Benth.) Brenan }\end{array}$ & $\begin{array}{l}\text { Anemocórica } \\
\text { Anemocórica } \\
\text { Anemocórica }\end{array}$ & $\begin{array}{c}- \\
3 \\
45\end{array}$ & $\begin{array}{c}76 \\
124 \\
177\end{array}$ \\
\hline $\begin{array}{l}\text { Lauraceae } \\
\text { Nectandra lanceolata } \mathrm{Nees} \\
\text { Nectandra megapotamica (Spreng.) Mez } \\
\text { Ocotea puberula (Rich.) Nees } \\
\text { O. pulchella (Nees) Mez }\end{array}$ & $\begin{array}{l}\text { Zoocórica } \\
\text { Zoocórica } \\
\text { Zoocórica } \\
\text { Zoocórica }\end{array}$ & $\begin{array}{c}18 \\
144 \\
320 \\
9\end{array}$ & $\begin{array}{c}- \\
275 \\
1 \\
10\end{array}$ \\
\hline $\begin{array}{l}\text { Malvaceae } \\
\text { Luehea divaricata Mart. \& Zucc. }\end{array}$ & Anemocórica & 2 & 192 \\
\hline $\begin{array}{l}\text { Myrtaceae } \\
\text { Campomanesia guazumifolia (Cambess.) O.Berg. } \\
\text { C. xanthocarpa O.Berg } \\
\text { Eucaliptus sp. } \\
\text { Eugenia ramboi D.Legrand } \\
\text { E. uruguayensis Cambess. }\end{array}$ & $\begin{array}{l}\text { Zoocórica } \\
\text { Zoocórica } \\
\text { Anemocórica } \\
\text { Zoocórica } \\
\text { Zoocórica }\end{array}$ & $\begin{array}{c}6 \\
6 \\
2 \\
2 \\
21\end{array}$ & $\begin{array}{l}- \\
- \\
- \\
-\end{array}$ \\
\hline $\begin{array}{l}\text { Pinaceae } \\
\text { Pinus elliottii Engelm. }\end{array}$ & Anemocórica & 8 & - \\
\hline $\begin{array}{l}\text { Primulaceae } \\
\text { Myrsine loefgrenii (Mez) Imkhan. } \\
\text { M. umbellata Mart. }\end{array}$ & $\begin{array}{l}\text { Zoocórica } \\
\text { Zoocórica }\end{array}$ & $\begin{array}{l}3 \\
-\end{array}$ & $\overline{4}$ \\
\hline $\begin{array}{l}\text { Rhamnaceae } \\
\text { Hovenia dulcis Thunb. }\end{array}$ & Zoocórica & 78 & 821 \\
\hline $\begin{array}{l}\text { Rosaceae } \\
\text { Prunus myrtifolia (L.) Urb. }\end{array}$ & Zoocórica & 118 & 2 \\
\hline $\begin{array}{l}\text { Rutaceae } \\
\text { Helietta apiculata Benth. }\end{array}$ & Anemocórica & 30 & 25 \\
\hline $\begin{array}{l}\text { Sapindaceae } \\
\text { Allophylus edulis (A.St.-Hil., Cambess. \& A. Juss.) Radlk. } \\
\text { A. guaraniticus (A. St.-Hil.) Radlk. } \\
\text { Allophyluspuberulus (Cambess.) Radlk. } \\
\text { Cupania vernalis Cambess. } \\
\text { Diatenopteryx sorbifolia Radlk. } \\
\text { Matayba elaeagnoides Radlk. }\end{array}$ & $\begin{array}{l}\text { Zoocórica } \\
\text { Zoocórica } \\
\text { Zoocórica } \\
\text { Zoocórica } \\
\text { Zoocórica } \\
\text { Zoocórica }\end{array}$ & $\begin{array}{c}229 \\
5 \\
524 \\
121 \\
73 \\
-\end{array}$ & $\begin{array}{c}904 \\
58 \\
166 \\
327 \\
5 \\
224\end{array}$ \\
\hline $\begin{array}{l}\text { Styracaceae } \\
\text { Styraxleprosus Hook. \&Arn. }\end{array}$ & Zoocórica & 38 & 40 \\
\hline Indeterminada 1 & Zoocórica & 3 & - \\
\hline
\end{tabular}



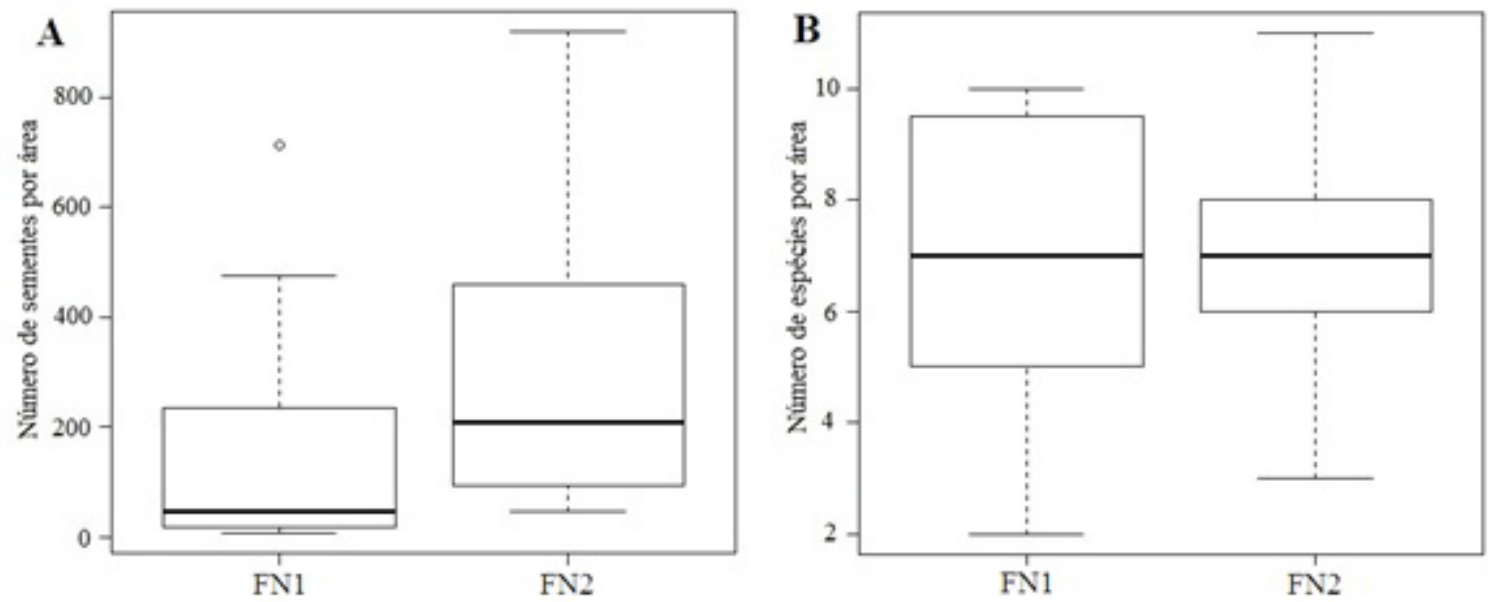

Figs. 1A, B. A. Número de sementes por área; B. número de espécies por área nos fragmentos de Floresta nativa 1 e Floresta nativa 2.

Tabela 3. Características estruturais e proporção de espécies e indivíduos totais amostrados de acordo com a estratégia de dispersão presentes na chuva de sementes nos fragmentos de Floresta nativa 1e Floresta nativa 2. $x^{2}=$ Qui-quadrado para uma amostra independente.

\begin{tabular}{|c|c|c|}
\hline Variáveis & Floresta nativa 1 & Floresta nativa 2 \\
\hline Abundância de sementes $\left(\mathrm{m}^{2}\right)$ & 1584 & 3445 \\
\hline Densidade de sementes $\left(\mathrm{m}^{2}\right)$ & $6.9 \pm 11.7$ & $14.6 \pm 25.3$ \\
\hline Riqueza de espécies & 28 & 21 \\
\hline Riqueza de famílias & 13 & 12 \\
\hline Diversidade de Shannon (nats. ind. ${ }^{-1}$ ) & 2.38 & 2.18 \\
\hline Equabilidade Pielou & 0.71 & 0.71 \\
\hline \multicolumn{3}{|l|}{ Indivíduos (\%) } \\
\hline Zoocóricos & 85 & 82 \\
\hline Anemocóricos & 14 & 17 \\
\hline Autocóricos & 1 & 1 \\
\hline$x^{2}$ & $122.6^{*}$ & $110.4^{*}$ \\
\hline \multicolumn{3}{|l|}{ Espécies } \\
\hline Zoocóricas & 61 & 67 \\
\hline Anemocóricas & 28 & 28 \\
\hline Autocóricas & 11 & 5 \\
\hline$x^{2}$ & $38.78^{*}$ & $58.9^{*}$ \\
\hline
\end{tabular}

Abundância de indivíduos, riqueza de espécies e de famílias calculadas a partir do número de indivíduos amostrados na área de estudo.* p $\leq 0.0001$.

\section{DISCUSSÃO}

Em pequenos fragmentos florestais, o processo de regeneração por meio da chuva de sementes, tende a ser responsável pela formação de uma comunidade semelhante a que está presente no momento. No presente estudo, observa-se que ambos os estratos arbóreos, adulto ou regenerante, são responsáveis pela proporção de sementes que chegam ao solo. Assim, percebe-se que além de contribuir com a sucessão no fragmento florestal, o estrato arbóreo regenerante também é responsável por contribuir com a chuva de sementes.

No fragmento florestal 1, caracterizado pela maior riqueza de espécies, observou-se uma relação positiva na composição do estrato arbóreo adulto e regenerante com a chuva de sementes. Apesar dos fragmentos estudados apresentarem um grande número de espécies, observamos que a riqueza total de espécies presente na chuva de sementes é menor em relação ao estrato arbóreo, assim como em outros trabalhos (Muller-Landau et al. 2002, Dalling \& John 2008). A menor riqueza de espécies na chuva de sementes pode estar associada ao fato que as sementes amostradas geralmente provêm de plantas que estão frutificando próximos aos coletores ou com copa acima do coletor (Hubbell 1979, Willson 1993).

Apesar da menor riqueza e diversidade na Floresta nativa 2 observou-se uma maior densidade de sementes neste fragmento. A presença das espécies Allophylus edulis (A.St.-Hil., Cambess. \& A. Juss.) Radlk. e da espécie exótica invasora Hovenia dulcis Thunb., contribuíram com um grande número de sementes, e podem ter sido responsáveis pela maior densidade de sementes apesar da menor riqueza observada neste fragmento.

Apesar da variação na riqueza de espécies, diversidade e abundância de sementes, não foi observada diferença entre as áreas estudas, pois ocorreu uma baixa variação 
na produção de sementes ao longo do ano. A maioria das espécies encontradas na chuva de sementes foi compartilhada entre os fragmentos florestais, fato que influenciou na ausência de diferença entre os fragmentos. Além disso, a presença de algumas espécies arbóreas abundantes na chuva de sementes e nos fragmentos florestais indica o importante papel ecológico dessas espécies na estrutura da comunidade florestal. Gaston (2011) ressalta que as espécies comuns são as mais importantes e alteram as funções e serviços ecossistêmicos. Na Floresta nativa 1, as espécies Allophylus puberulus, Ocotea puberula e A. edulis, foram responsáveis por $67,5 \%$ do total de sementes amostradas na chuva de sementes, enquanto na Floresta nativa 2, as sementes das espécies $A$. edulis, $H$. dulcis e Cupania vernalis, corresponderam a $59,5 \%$ do total amostrado.

A maioria das espécies amostradas na chuva de sementes é zoocórica em ambos os fragmentos, sendo o padrão observado em florestas na região sul-sudeste do Brasil (Caldato et al. 1996, Budke et al. 2005, Rother 2006, Campos et al. 2009, Capellesso et al. 2013, Capellesso et al. 2015), confirmando a importância da fauna silvestre para a dispersão das espécies arbóreas. Conclui-se que a fauna apresenta grande importância na dinâmica florestal, influenciando a regeneração das populações e atuando na conservação de pequenos fragmentos florestais de Mata Atlântica no Sul do Brasil (Tabarelli \& Peres 2002).

As sementes são dispersas pela fauna em novas áreas quando o seu dispersor é atraído até esses locais (Ceccon et al. 2006). Todavia, uma alta proporção de sementes pode permanecer armazenada no banco de sementes na própria área onde os frutos foram produzidos. Em razão disso, uma importante estratégia para os processos de regeneração e conservação de pequenos fragmentos florestais é a manutenção da conectividade e a conservação do entorno que possibilita o deslocamento dos animais e, consequentemente, a dispersão das sementes. Costa et al. (2012), observaram que a dispersão pode ser afetada diretamente pela fragmentação dessas áreas, onde fragmentos menores e sem conectividade podem dificultar o movimento dos dispersores.

Os resultados observados permitem inferir, a partir da chuva de sementes, que o processo de regeneração está associado com a estrutura da vegetação nos fragmentos florestais. O fragmento com maior diversidade (Floresta nativa 1) apresentou uma estreita relação entre o estrato arbóreo adulto e regenerante com a chuva de sementes, o que pode sugerir que este fragmento apresenta um estádio de regeneração mais avançado em relação a Floresta nativa 2. Por outro lado, na Floresta nativa 2, que apresenta uma forte influência da espécie exótica invasora $H$. dulcis na produção de sementes, não apresentou relação com o estrato arbóreo adulto, sugerindo que essa área está em processo de estruturação, com os indivíduos do estrato arbóreo regenerante apresentando uma relação direta com a chuva de sementes e, consequentemente com a regeneração. É importante salientar que a riqueza dos estratos arbóreo adulto e regenerante não foram semelhantes entre as duas áreas, demonstrando que a diversidade na composição florística se manifesta em pequenos fragmentos no Sul do Brasil.

\section{REFERÊNCIAS}

Budke, J.C., Athayde, E.A., Giehl, E.L.H., Záchia, R.A., Eisinger, S.M. 2005. Composição florística e estratégias de dispersão de espécies lenhosasem uma floresta ribeirinha, arroio Passo das Tropas, Santa Maria, RS, Brasil. Iheringia, Série Botânica, 60(1):17-24.

Caldato, S.L., Floss, P.A., Croce, D.M. \& Longhi, S.J. 1996. Estudo da regeneração natural, banco de sementes e chuva de sementes na reserva genética florestal de Caçador, SC. Ciência Florestal 6:27-38.

Campos, E.P., Vieira, M.F., Silva, A.F., Martins, S.B., Carmo, F.M.S. Moura, V.M. \& Ribeiro, A.S.S. 2009. Chuva de sementes em Floresta Estacional Semidecidual em Viçosa, MG, Brasil. Acta botanica brasilica 23(2):451-458.

Capellesso, E.S., Sganzerla, F.L., Santolin, S.F., Dariva, G. \& Zanin, E.M. 2013. Banco e chuva de sementes em fragmento florestal urbano no Sul do Brasil. Perspectiva 37(137):123-132.

Capellesso, E.S., Santolin, S. F. \& Zanin, E.M. 2015. Banco e chuva de sementes em área de transição florestal no Sul do Brasil. Revista Árvore 39(5):821-829.

Capellesso, E.S., Scrovonski, K.L., Zanin, E.M., Hepp, L.U., Bayer, C., Sausen, T.L. 2016. Effects of forest structure on litter production, soil chemical composition and litter-soil interactions. Acta Botanica Brasilica 30(3):329-335.

Ceccon, E., Huante, P. \& Rincon, E. 2006. Abiotic factors influencing tropical dry forests regeneration. Brazilian Archives of Biology and Technology 49:305-312.

Clark, J.S., Fastie, C., Hurtt, G., Jackson, S.T., Johnson, C., King, G.A., Lewis, M., Lynch, J., Pacala, S., Prentice, C., Schupp, E.W., Webb III, T. \& Wyckoff, P. 1998. Reid's paradox of rapid plant migrationdispersal theory and interpretation of paleoecological records. Bioscience 48:13-24.

Costa, J.B.P., Melo, F.P.L., Santos, B.A. \& Tabarelli, M. 2012. Reduced availability of large seeds constrains Atlantic forest regeneration. Acta Oecologica 39:61-66.

Dalling, J.W. \& John, R. 2008.Seed limitation and the coexistence of pioneer tree species. In Tropical Forest Community Ecology (W.P. Carson, S.A. Schnitzer). Wiley-Blackwell, Oxford, p. 242-253. 537p.

Decian, V., Zanin, E.M., Henke, C., Quadros, F.R. \& Ferrari, C.A. 2009. Uso da terra na região Alto Uruguai do Rio Grande do Sul e obtenção de um banco de dados relacional de fragmentos de vegetação arbórea. Perspectiva 33(121):165-176.

Fahrig, L. 2003. Effects of habitat fragmentation on biodiversity. Annual Review of Ecology, Evolution, and Systematics 34:487-515.

Gaston, K.J. 2011.Common Ecology. BioScience 61(5):354-362.

Hardesty, B.D. \& Parker, V.T. 2003. Community seed rain patterns and a comparison to adult community structure in a West African tropical forest. Plant Ecology 164:49-64.

Hubbell, S.P. 1979. Tree dispersion, abundance, and diversity in a tropical dry forest. Science 203(4387):1299-1309.

Laurance, W.F., Nascimento, H.E.M., Laurance, S.G., Andrade, A.C., Fearnside, P.M.,

Ribeiro, J.E.L. \& Capretz, R.L. 2006. Rain forest fragmentation and the proliferation of successional trees. Ecology 87:469-482.

Martínez-Garza, C., Osorio-Beristain, M., Valenzuela-Galván, D. \& Nicolás-Medina, A. 2011. Intra and inter-annual variation in seed rain in a secondary dry tropical forest excluded from chronic disturbance. Forest Ecology and Management 262:2207-2218.

Muller-Landau, H.C., Wright, S.J., Calederón, O., Hubbell, S.P. \& Foster, R.B. 2002.Assessing recruitment limitation: Concepts, methods, and case studies from a tropical forest. In Seed Dispersal and Frugivory: Ecology, Evolution, and Conservation (D.J. Levey, W.R. Silva \& M. Galetti). CAB International, Oxon, UK. 511p.

Myers, N., Mittermeier, R.A., Mittermeier, C.G., Fonseca, G.A.B. \& Kent, J. 2000.Biodiversity hotspots for conservation priorities. Nature 403:853-858.

Oliveira-Filho, A.T., Budke, J.C., Jarenkow, J.A., Eisenlohr, P.V. \& Neves, D.R.M. 2013. Delving into the variations in tree species composition 
and richness across South American subtropical Atlantic and Pampean forests. Journal of Plant Ecology 2:1-23.

Pietro-Souza, W., Silva, N.M. \& Campos, E.P. 2014. Chuva de sementes em remanescentes florestais de Campo Verde, MT. Revista Árvore 38(4):689-698.

Rother, D.C. 2006. Chuva de sementes e estabelecimento de plântulas em ambientes com bambus na Mata Atlântica. 123f. Dissertação de Mestrado, Universidade Estadual Paulista, Rio Claro.

Simpson, R.L. 1989. Ecology of soil seed bank, Academic Press, San Diego.

Sobral, M., Jarenkow, J.A., Brack, P., Irgang, B., Larocca, J. \& Rodrigues, R.S. 2013. Flora arbórea e arborescente do Rio Grande do Sul, Brasil. RiMA/Novo Ambiente, São Carlos.

SOS Mata Atlântica. 2015. Atlas dos remanescentes florestais da Mata Atlântica 2013-2014. Disponível em: https://www.sosma.org.br/ projeto/atlas-damata-atlantica. Acesso em dezembro de 2016.
Tabarelli, M. \& Peres, C.A. 2002. Abiotic and vertebrate seed dispersal in the Brazilian Atlantic Forest: implications for forest regeneration. Biological Conservation 106:165-176.

Team, R. Core. 2012. R: a language and environment for statistical computing. Vienna: R Foundation for Statistical Computing, $p$. 618-622.

Terborgh, J. 1986. Keystone plant resources in the tropical forest. In Conservation Biology: the Science of Scarcity and Diversity (M.E. Soulé). Sinauer, Sunderland, p. 330-344.

Van Der Pijl, L. 1982. Principles of dispersal in higher plants. Springer Verlag, New York. 218p.

Vieira, D.L.M. \& Scariot, A. 2006. Principles of natural regeneration of tropical dry forests for restoration. Restoration Ecology 14:11-20.

Willson, M.F. 1993. Dispersal mode, seed shadows, and colonization patterns. Vegetation 107/108:261-208. 